\title{
Lifted Bottom of Microfluidic Channel for Cell Absorption Spectroscopy Using Optical Fibers
}

\author{
Motohide Yoshimi, Shinya Kumagai, Yasutake Ohishi, and Minoru Sasaki* \\ Toyota Technological Institute, 2-12-1 Hisakata, Tenpaku-ku, Nagoya 468-8511, Japan
}

(Received August 8, 2016; accepted January 19, 2017)

Keywords: absorption spectroscopy, cell, optical fiber, bias spring, lifted bottom

A Si microoptical bench has been developed for measuring the absorption spectrum of cells in suspension. Optical fibers are the elements for constructing a light path inside a Si chip. The sensing light is introduced, transmitted through the sample, and detected. The light propagates in the core. A thick surrounding cladding protects the beam from optical disturbance. In many cases, the cells in suspension tend to sink to the bottom of the microfluidic channel because their density is higher than that of water. For bringing the cell in the sensing light beam, the bottom of the microfluidic channel is lifted up to the core height of the optical fiber in the device. The absorption caused by the chlorophyll of the chlorella is observed.

\section{Introduction}

The microfluidic devices in micrototal analysis systems (mTAS) handle a small amount of liquid in a well-controlled and material-saving manner. Biological samples can be in the liquid. Their analysis is mainly based on chemical methods. As for living samples, such as cells in suspension, applying a chemical reaction often involves a nonreversible reaction, which disturbs bioactivity. Optical spectroscopy is known to be noninvasive and informative. ${ }^{(1,2)}$ The activity of a single cell encapsulated in a droplet can be detected by the fluorescence related to secreted proteases. ${ }^{(3,4)}$ The bulk microscopes are combined for dealing with the light. The main advantage using the optical fiber for illuminating the sample in the microfluidic channel is the miniaturization and the integration of the optical function. Light is guided in an optical fiber, which consists of a core and a cladding. The cladding protects the guided light in the core from disturbance. Thus, once the fibers are mechanically set, a functional combination is expected.(5,6) Measuring absorbance spectra is widely used in common macro-structure sensing. At present, supercontinuum light and a frequency comb are attractive light sources. ${ }^{(7-9)}$ Poly(dimethylsiloxane) is used for integrating the microchannel and embedding the fibers; however the simple setting means low alignment accuracy and limited illumination efficiency. ${ }^{(10)}$ Many cells are heavier than water. The relative density of red blood cells is about 1.1, and they sink after centrifugal separation. Many aquatic plants also tend to sink in water. This requires specific care in microfluidic design for irradiating sensing light on a sample. An optical trap is applied to align the single cell in the optical axis. Improvement of the repeatability and stability is obtained at the cost of the additional fiber laser and the complicated setup. ${ }^{(11)}$ In this study, a new Si microoptical bench with a robust basement is proposed to measure the absorption spectrum of cells in liquid.

${ }^{*}$ Corresponding author: e-mail: mnr-sasaki@toyota-ti.ac.jp http://dx.doi.org/10.18494/SAM.2017.1447 


\section{Device}

\subsection{Cell sample}

The chlorella cells used in this study have a spherical shape. This average diameter is $6.3 \pm 0.4$ $\mu \mathrm{m}$ (number of samples: 30 ). The number density is about $2800 \mathrm{cells} / \mathrm{ml}$, corresponding to one cell in a cube having an edge length of $710 \mu \mathrm{m}$. Chlorella contains chlorophyll, which is known to have absorptions at 400-500 and 650-700 nm wavelengths. The chlorella cells used are slightly heavier than water, and thus they gradually sink to the bottom of the bottle, as shown in Fig. 1(a).

\subsection{Design}

Figure 1(b) shows the lateral design of the device, which consists of optical fibers ${ }^{(12)}$ and a microfluidic channel. Two optical fibers are allowed to face each other. Between these fibers, a microfluidic channel passes. The device layer thickness is designed to be $125 \mu \mathrm{m}$, which is the same as the optical fiber diameter. Figure 1(c) shows the conventional vertical design. A fiber cladding makes the optical beam position higher than the trench bottom. Thus, the sensing light irradiation on the cells has a low possibility. Since the cell sample should be in the light path as

(a)

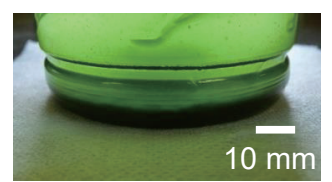

(b)

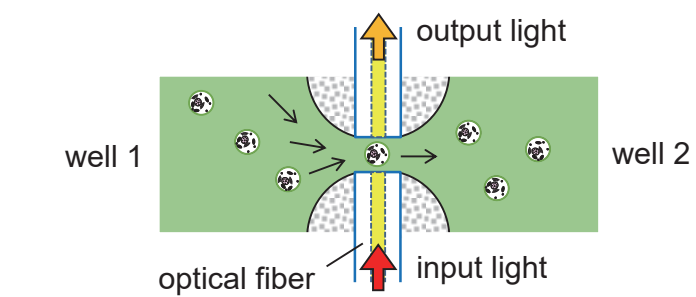

(c)

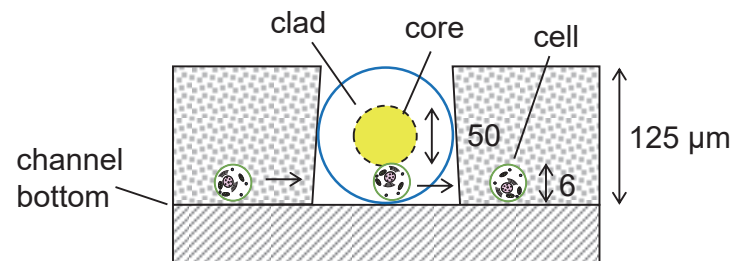

(d)

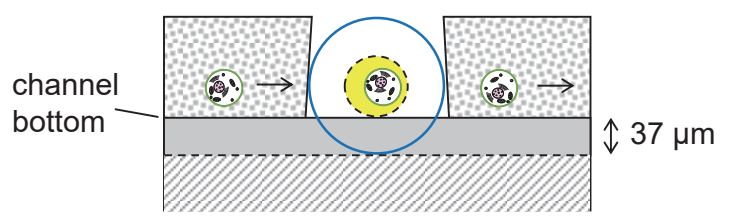

Fig. 1. (Color online) (a) Chlorella cells sunk in suspension. (b) Lateral design combining the microfluidic channel and the optical fibers. Vertical design (c) without and (d) with the lifted bottom of the microfluidic channel. 
much as possible, it is better for the fiber core to be near the floor of the microfluidic channel. Figure 1(d) shows the proposed design. The optical fiber used is multimode with a core diameter of $50 \mu \mathrm{m}$. The cladding thickness is $37.5 \mu \mathrm{m}$. The bottom of the microfluidic channel is $37 \mu \mathrm{m}$ higher than the trench bottom for setting the fiber. The channel width is smaller than the fiber core to maintain the optical transmission rate.

\subsection{Bias spring}

For obtaining a high transmission ratio of the sensing light through the gap, two fibers should be aligned for accuracy. ${ }^{(13)}$ The single patterning guarantees the straightness of the fiber guide at the mask level. The bias spring included in the trench pushes the fiber laterally to the opposite sidewall. When the sidewall is tapered in reverse, even though the angle is small, it can push the fiber both laterally and vertically. ${ }^{(14)}$ This increases the alignment accuracy. There are 15 bias springs for one fiber.

\section{Fabrication}

Figure 2 shows the fabrication sequence. The starting material is a silicon-on-insulator (SOI) substrate (the device and buried oxide layer thicknesses are 125 and $1 \mu \mathrm{m}$, respectively) with an oxide cover. The embedded mask technique is used. (1) A photoresist is spin-coated. This film is patterned and UV-cured. (2) The oxide cover is removed by etching. Again, the photoresist is spincoated and patterned. (3) Using the top photoresist mask, deep reactive ion etching (RIE) is carried out. Next, $\mathrm{O}_{2}$ ashing is performed to remove the $\mathrm{C}_{x} \mathrm{~F}_{y}$ film that is deposited during the previous

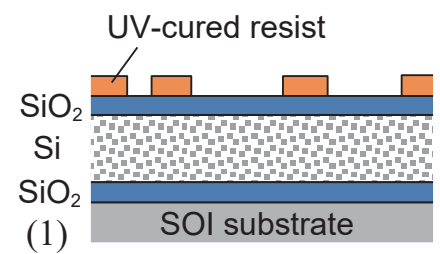

(4)

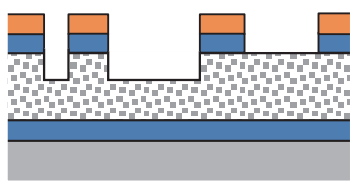

(2)

resist

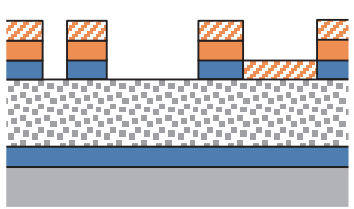

(3)

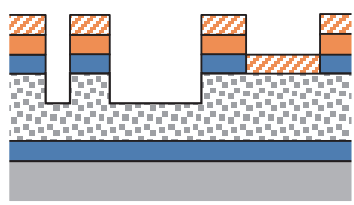

(6)

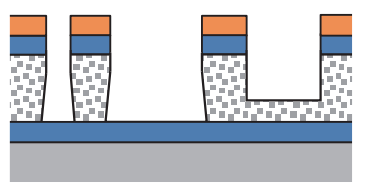

(5)

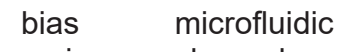

spring channel

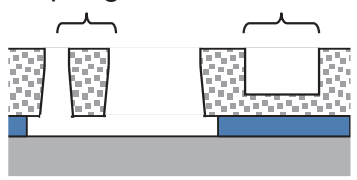

Fig. 2. (Color online) Fabrication sequence. 
deep RIE. (4) Then, the top photoresist is flush-exposed and developed to be removed. The embedded UV-cured photoresist mask appears. (5) A second deep RIE is carried out. The etching time after the deeper position has reached the buried oxide is adjusted to control the bottom height of the microfluidic channel. The Si etching profile is slightly negative, giving a reversed taper in the sidewall. (6) The buried oxide layer is etched using concentrated HF to release the bias springs. The structure is dried in air after rinsing with water.

Figure 3(a) shows a wide view of the Si chip. A $136 \mu \mathrm{m}$ wide trench with a bias spring is used for setting the optical fiber. A channel with a narrow width at the center is designed to flow the liquid sample. The bottom is higher than that of the trench for the optical fiber, as shown in Fig.

(a)

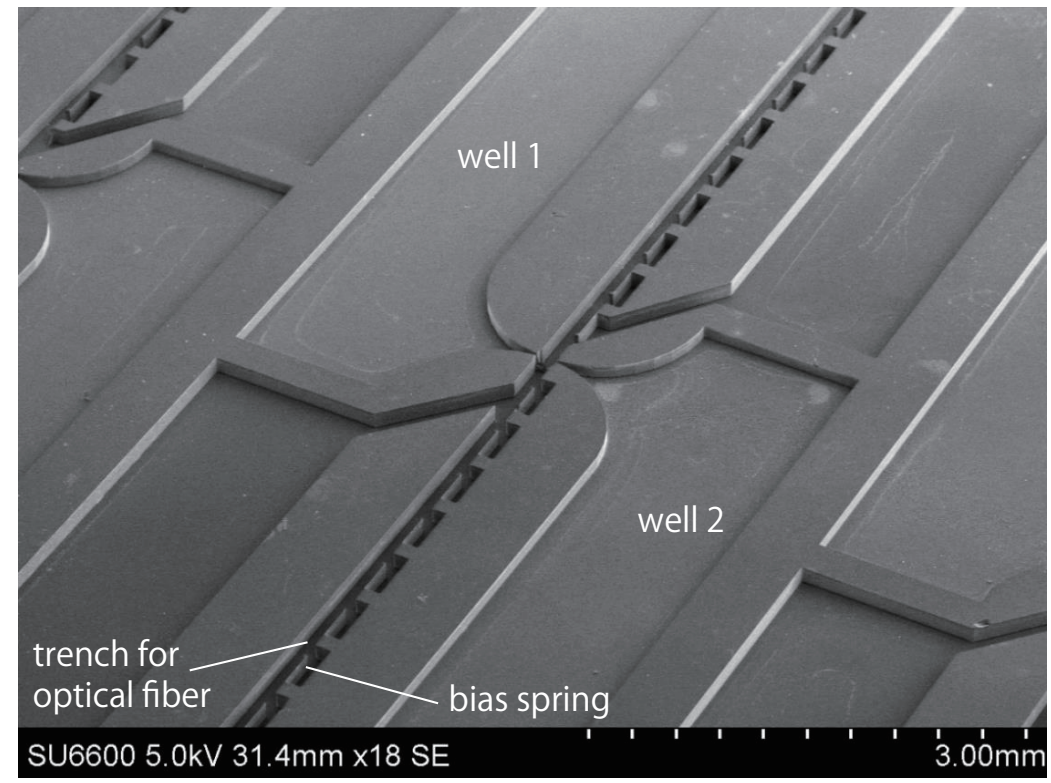

(b)

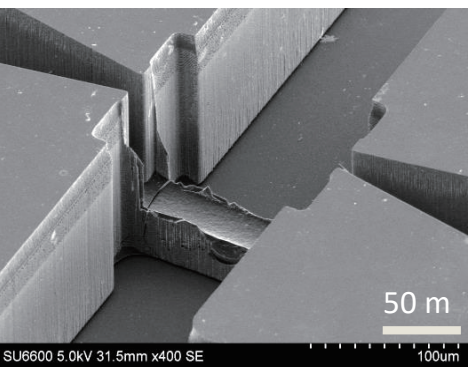

(d)

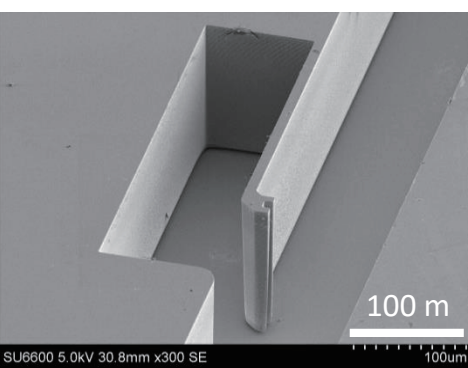

(c)

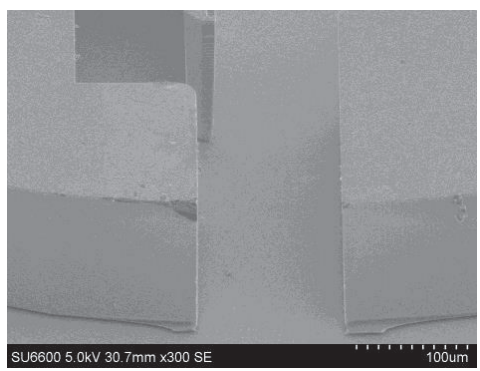

(e)

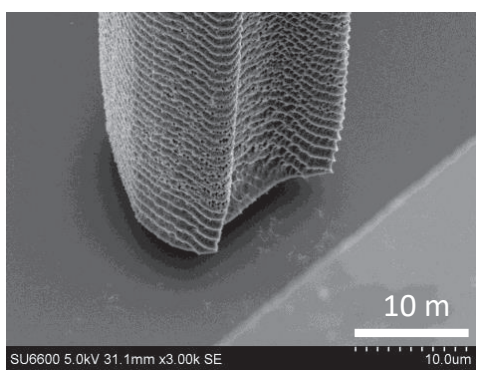

Fig. 3. SEM images of the device. (a) Wide view. (b) Crosspoint of the microfluidic channel and trench for the optical fiber. (c) Cross-sectional view of the trench. (d) Bias spring. (e) Released bottom of the bias spring. Images (c)-(e) were obtained from the test wafer. 
3(b). Figure 3(c) shows a cross-sectional view of the trench. A negative profile can be seen. The reverse taper is about $0.6^{\circ}$. Figure $3(\mathrm{~d})$ shows the bias spring. The end of the contact finger has a parallelogram shape. The bias spring easily deflects when the optical fiber is inserted from the outside. Figure 3(e) shows the bottom of the bias spring, indicating the release of the spring without adhesion.

Figure 4 shows the profile of the device measured using a white-light interferometer (Zygo, NewView 7300). The height of the microfluidic channel from the fiber trench is $37 \mu \mathrm{m}$, which agrees well with the design value. The channel width is $25 \mu \mathrm{m}$.

\section{Experiments and Results}

A suspension with chlorella cells is poured in the wells [shown in Fig. 3(a)]. Figure 5(a) shows an optical image. The tiny dots correspond to the chlorella cells. When one well has cotton for vaporizing water, flow occurs from the opposite well. The cells flow through the gap between

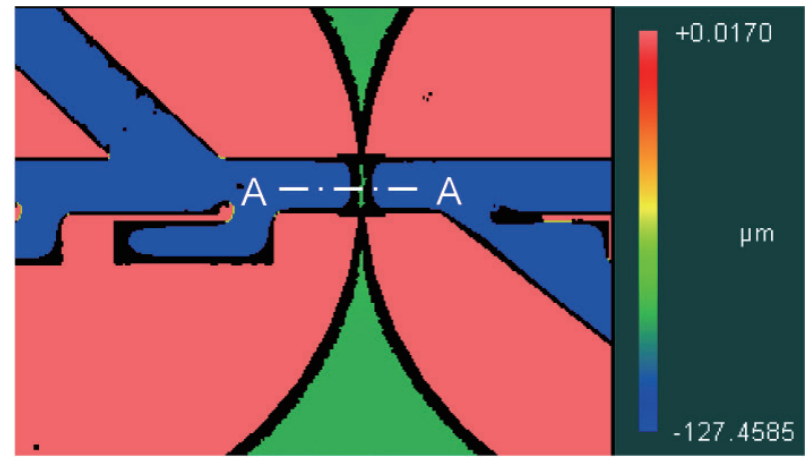

(a)

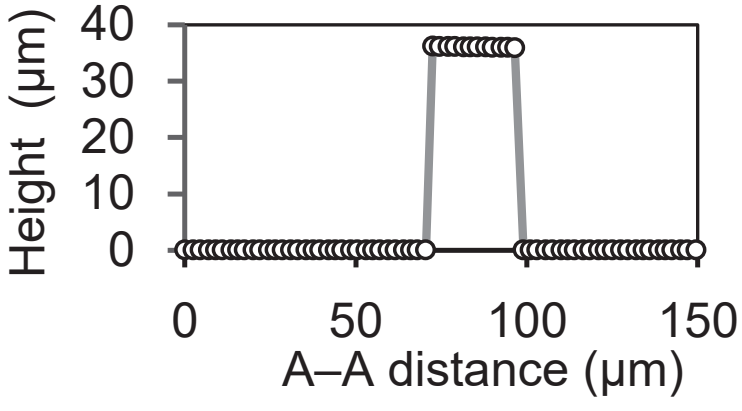

(b)

Fig. 4. (Color online) (a) Height map of the device. The black shadow indicates the area with little measurement information although a Si structure exists. (b) Cross-sectional profile at the microfluidic channel across A-A in (a).

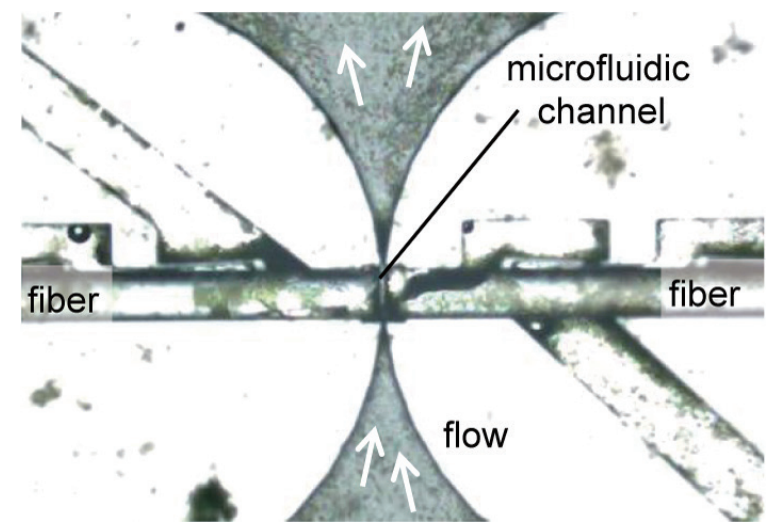

(a)

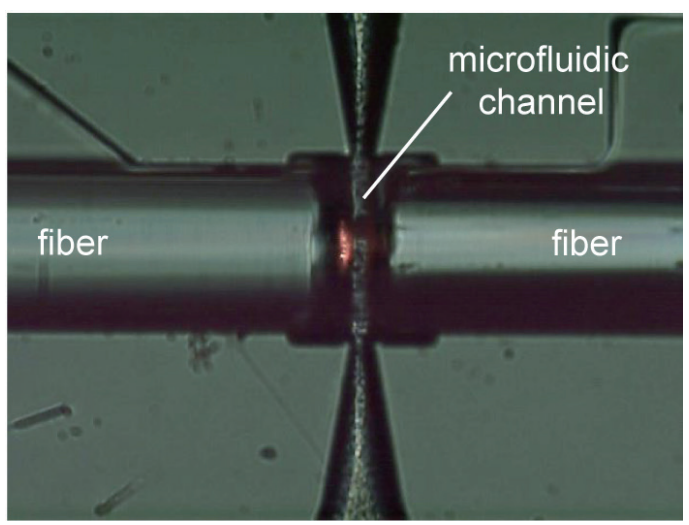

(b)

Fig. 5. (Color online) (a) Optical micrograph confirming flow through the gap between fibers. (b) Magnified image showing the sensing light irradiation from the left fiber. 


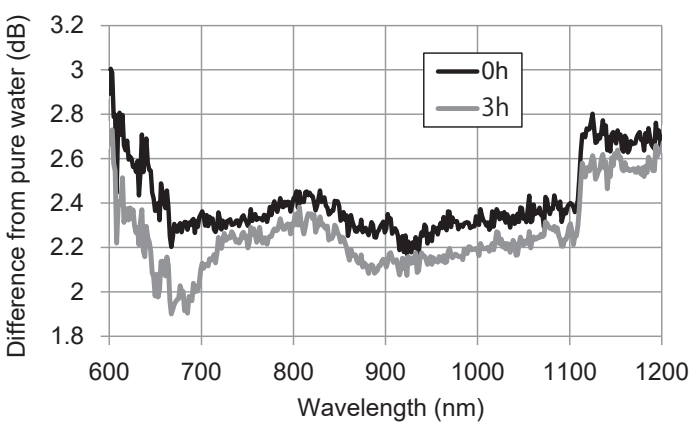

(a)

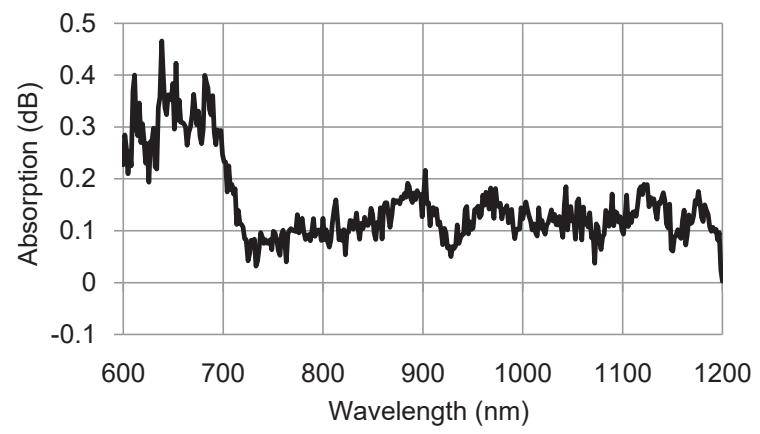

(b)

Fig. 6. Measured transmission spectra of the chlorella suspension. (a) The differences from pure water obtained at 0 and $3 \mathrm{~h}$ after the suspension is introduced. Transmission intensity increases slightly from that of pure water, as the index matching of the suspension between the optical fiber becomes better. (b) Absorption spectrum of chlorella.

the fibers. Figure 5(b) shows the magnified image around the fiber gap. The bright area indicates illumination by the light guided through the fiber. The light source is a halogen lamp (Anritsu, MG922A).

Figure 6(a) shows the transmission spectra observed using an optical spectrum analyzer (Anritsu, MS9780A). The data are the differences from the values obtained from pure water. The black curve is observed just after the suspension with chlorella cells is introduced. The cells drift in the microfluidic channel with minimal light irradiation. Then, the chlorella cells gradually sink and accumulate on the bottom of the microfluidic channel. After $3 \mathrm{~h}$, the gray curve is observed. The spectra are similar to each other except for the absorption around $680 \mathrm{~nm}$, which is related to the chlorophyll. Figure 6(b) is the absorption spectrum showing the differences between these two spectra. Compared with the density gradient media $\left(O p t i P r e p^{\mathrm{TM}}\right)$ known for suspending the organelles at a specific height, ${ }^{(15)}$ the lifted bottom is simple and effective for all cells which move to the bottom. Although this device needs time to allow the cells to sink in the optical path, an additional function can be added to attract the cells electrically to the bottom of the microfluidic channel; this will be included in a future study.

\section{Conclusions}

A microfluidic channel and optical fibers are assembled in a Si microoptical bench. The height of the microfluidic channel is lifted for measuring the absorption spectra of the cells, which tend to sink because their density is higher than that of water. The Si etching depth and the straightness of the fiber guide trenches are well controlled. The chlorella cells are introduced into the gap between two fibers and their absorption due to the chlorophyll is observed.

\section{Acknowledgements}

This study was supported by MEXT program for Forming Strategic Research Infrastructure (S1101028) Japan. 


\section{References}

1 B. Kuswandi, Nuriman, J. Huskens, and W. Verboom: Analytica Chimica Acta 601 (2007) 141.

2 J. T. Adeosun and A. Lawal: Sens. Actuators, B 139 (2009) 637.

3 E. X. Ng, M. A. Miller, T. Jing, and C.-H. Chen: Biosens. Bioelectron. 81 (2016) 408.

4 T. Jing, R. Ramji, M. E. Warkiani, J. Han, C. T. Lim, and C.-H. Chen: Biosens. Bioelectron. 66 (2015) 19.

5 C. L. Hagen, J. R. Schmidt, and S. T. Sanders: IEEE Sensors J. 6 (2006) 1227.

6 A. Garcia-Uribe, K. C. Balareddy, J. Zou, and L. V. Wang: IEEE Sensors J. 8 (2008) 1698.

7 D. Deng, L. Liu, T. H. Tuan, Y. Kanou, M. Matsumoto, H. Tezuka, T. Suzuki, and Y. Ohishi: J. Ceram. Soc. Jpn. 124 (2016) 103.

8 L. Liu, K. Nagasaka, G. Qin, T. Suzuki, and Y. Ohishi: Appl. Phys. Lett. 108 (2016) 011101.

9 W. Gao, M. Liao, D. Deng, T. Cheng, T. Suzuki, and Y. Ohishi: Opt. Commun. 300 (2013) 225.

10 M. L. Chabinyc, D. T. Chiu, J. C. McDonald, A. D. Stroock, J. F. Christian, A. M. Karger, and G. M. Whitesides: Anal. Chem. 73 (2001) 4491.

11 W. Z. Song, A. Q. Liu, S. Swaminathan, C. S. Lim, P. H. Yap, and T. C. Ayi: Appl. Phys. Lett. 91 (2007) 223902.

12 C. Marxer, C. Thio, M.-A. Gretillat, N. F. de Rooij, R. Battig, O. Anthamatten, B. Valk, and P. Vogel: J. Microelectromech. Syst. 6 (1997) 277.

13 K. Kawano: Fundamentals and Applications of Optical Coupling System for Optical Device (Gendaikougakusha, Tokyo, 1994) Chap. 4 (in Japanese).

14 H. Iimura, D. Deng, Y. Ohishi, S. Kumagai, and M. Sasaki: JPS Conf. Proc. 1 (2014) 015071.

15 E. Gias, S. U. Nielsen, L. A. F. Morgan, and G. L. Toms: J. Virol. Methods 147 (2008) 328.

\section{About the Authors}

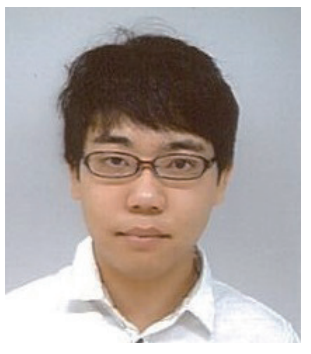

Motohide Yoshimi received his B.S. degree from Toyota Technological Institute, Japan, in 2016. He is currently an M.S. student.

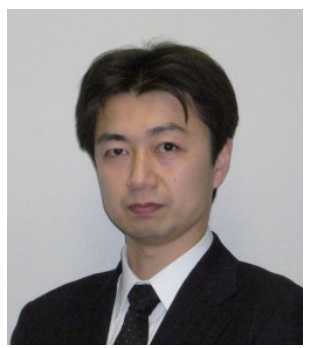

Shinya Kumagai received his M.S. and Dr. Eng. degrees from Tohoku University, Japan, in 1998 and 2001, respectively. From 2001 to 2004, he was a research associate at the Institute of Fluid Science, Tohoku University. In 2004, he joined the Advanced Technology Research Laboratories, Matsushita Electric Industrial Co., Ltd. Since 2008, he has been an associate professor at Toyota Technological Institute. His current interest is bionanotechnology combined with micro/nanofabrication processes.

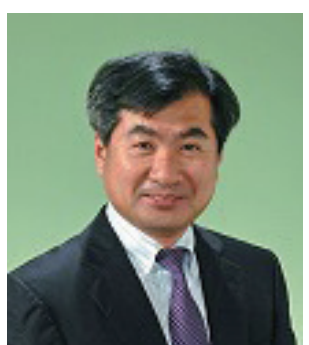

Yasutake Ohishi received his M.S. degree from Tohoku University, Japan, in 1980. From 1980 to 1989 , he was a member of the NTT Electrical Communications Laboratories. He received his Dr. Eng. degree from Tokyo Institute of Technology in 1988. From 1989 to 1990, he was a visiting scientist at Rutgers University, USA. From 1990, he was with the NTT Opto-Electronics Laboratories. From 2002, he was with the NTT Basic Research Laboratories. Since 2002, he has been a professor at Toyota Technological Institute, and since 2013, he has been the vice president there. His research is on photonics materials and devices, especially related to optical fibers. 


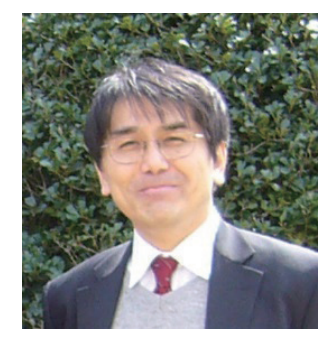

Minoru Sasaki received his M.S. and Dr. Eng. degrees from Nagoya University in 1993 and 1995, respectively. In 1995, he was a Research Fellow of the Japan Society for the Promotion of Science. In 1996, he joined the Department of Mechatronics and Precision Engineering, Tohoku University. Since 2007, he has been a professor at Toyota Technological Institute. His current research includes MEMS sensors and microfabrication, especially related to 3D structures. 